\title{
Interplay between Exercise and SIRT1 in Skeletal Muscle of Diabetic Male Rats
}

\author{
ASMAA S. MOHAMMED, M.D.*; NERMEEN B. SADEK, M.D.*; MONA M. MOHAMMED, M.D.*; \\ EFFAT A. KHOWAILED, M.D.* and LAILA A. RASHED, M.D.
}

The Departments of Medical Physiology* and Biochemistry**, Faculty of Medicine, Cairo University

\begin{abstract}
Background: Great evidences support the promising effect of physical exercise on pathophysiological changes associating diabetes. Several molecular mechanisms of the beneficial outcome of physical training have been suggested. The role of silent information regulator one (SIRT 1) in diabetes after exercise training is still obscure.

Aim of Study: To evaluate wheather re-establishing SIRT 1 activity after exercise training can improve diabetic outcome or not.

Patients and Methods: Forty male Wistar albino rats ( $\mathrm{n}=10$ per group) were randomized to control, diabetic (STZ; $60 \mathrm{mg} / \mathrm{kg}$ i.p.), diabetes + exercise (D+EX) and exercise + diabetes $(\mathrm{EX}+\mathrm{D})($ swimming $1 \mathrm{~h} /$ day, 5days/week before diabetes). After the end of the study, body mass index (BMI), serum glucose, triglyceride, HDL, SIRT1 expression and activity were measured.

Results: Both exercised groups showed significant decrease in serum glucose and triglycerides, significant increase in serum HDL, SIRT1level and activity and insignificant change in BMI compared with control group. There was no significant difference between exercised groups, approaching to normal levels in EX+D group. Correlation studies showed SIRT1 level and activity negatively correlated with glucose and TG levels, positively correlated with HDL level among all groups.

Conclusion: Exercise training before or after diabetes improved glycemic control and lipid profile, SIRT upregulation may have a role in this improvement.
\end{abstract}

Key Words: Diabetes - Exercise training - SIRT level - SIRT activity.

\section{Introduction}

DIABETES has become a widespread epidemic that threats human health over the past four decades [1]. Skeletal muscle represents $40 \%$ of body mass and plays an important role in the maintenance of metabolic health. Healthy skeletal muscle is sensitive to insulin and efficiently stores glucose [2] .

Correspondence to: Dr. Asmaa S. Mohammed,

E-mail: sama.selmy@yahoo.com
Plasticity of skeletal muscle plays a great role in the remodeling of metabolic function towards a healthy phenotype in all populations, for example, exercise training can significantly improves skeletal muscle mitochondrial function and metabolic health [3].

Several public health recommendations recognize that regular exercise has a fundamental role in the prevention, and treatment of numerous chronic conditions including obesity and type 2 diabetes mellitus (T2DM) [4].

Therefore, exercise, as one of lifestyle intervention, may be an alternative to other pharmacological interventions [5].

Exercise-induced changes in skeletal muscle have been implicated in the activation of signal transduction pathways regulating skeletal muscle plasticity. One of these pathways is related to the increased activity of a nuclear protein, SIRT1 [4] Sirtuin 1 (SIRT1) is a mammalian homolog of the yeast protein silent information regulator 2 (Sir2), which depends on NAD (nicotinamide adenine dinucleotide) for its activity. Its role is through deacetylating a variety of substrates, including histones, transcription factors and co regulators, to regulate target gene expression in stress responses and cellular metabolism [6].

In humans, it regulates different metabolic processes including hepatic lipid metabolism and gluconeogenesis, pancreatic insulin secretion, fat cell accumulation and maturation, as well as circadian regulation of metabolism [7].

SIRT1 activators improve glucose homeostasis and insulin sensitivity in adipose tissue, skeletal muscle and liver [6]. Thus, SIRT1 activation may 
be a promising new therapeutic approach for treating diseases of ageing such as type 2 diabetes.

It is still unclear whether the effect of exercise in modulation of SIRT activity will be more beneficial before or after diabetes.

Therefore, The present study was undertaken to test the hypothesis that exercise training reestablishes physiologically relevant activity of SIRT1, which has been attenuated with diabetes, and compare between the effect of exercise before (as prophylactic measure) and after (as curative measure) induction of diabetes.

\section{Patients and Methods}

\section{Experimental animals:}

The study was carried out on forty male Wistar rats $(160-200 \mathrm{~g})$. The animals were purchased and housed in the Experimental Animal Unit, Faculty of Medicine, Cairo University in 2014, under constant temperature (25C), relative humidity $(60 \pm 5 \%)$, and 12-h light/dark cycle. Rats were kept in wire mesh cages and had free access to food and water throughout the experimental period.

\section{Experimental design:}

After acclimatization, rats were randomly divided into 4 groups $(\mathrm{n}=10$ each). Control group received a single intraperitoneal (i.p.) injection of $0.1 \mathrm{M}$ sodium citrate buffer $(\mathrm{pH} 4.5)$. The remaining 30 rats received an intra-peritoneal injection of streptozotocin (STZ) to induce Type 1 diabetes $(60 \mathrm{mg} / \mathrm{kg}$, dissolved in $0.01 \mathrm{M}$ citrate buffer, $\mathrm{pH}=4.5$; Sigma; St. Louis, Mo, USA) [8,9]. Diabetes was confirmed by measuring fasting serum glucose 3 days after STZ injection. These diabetic rats further subdivided into diabetic group (DM), remain sedentary without exercise till the end of the work, post diabetes exercise group (DM + Ex) undergo exercise for 1 month after induction of diabetes and pre diabetes exercise $(\mathrm{Ex}+\mathrm{DM})$ undergo exercise for 1 month before induction of diabetes.

All experimental procedures were conducted according to the guide for the care and use of laboratory animals published by the US National Institute of Health (NIH, 1985), and approved by the local ethical and scientific committee (Faculty of Medicine, Cairo University).

\section{Experimental procedures:}

Exercise protocol:

The trained groups were first adapted to the water environment. Adaptation was performed over 10 days in the same tank where the training was performed to reduce animal stress and to avoid possible physiological adaptations that might improve the physical capacity of the animals [10]

Rats were placed in shallow water for $10 \mathrm{~min}$ for three days. The water depth was then increased gradually. By the fourth day, animals swam for 5 min in deep water. The length of time was increased by $10 \mathrm{~min}$ each day until the 10 th day of adaptation [10]. Then the rats' swam in individual tanks that contained water for $1 \mathrm{~h}$ per day, 5 days per week for one month [11]

\section{Body mass index (BMI) measurement:}

Rats were weighted in grams \& their naso-anus lengths in $\mathrm{cm}$ were measured at the end of the work [12].

The obesity index was calculated according to an equation formulated by [13] : BMI=weight in grams/naso anal length in $\mathrm{cm}^{2}$.

\section{Biochemical assessment:}

At the end of the study, all rats were subjected to overnight fasting, blood samples were collected from the retro-orbital plexus for the assessment of fasting serum glucose, insulin, triglycerides (TGs) and high density lipoprotein (HDL) levels. Then the rats were euthanized and the soleus muscle was rapidly dissected out and frozen in liquid nitrogen and stored at $80^{\circ} \mathrm{C}$ for measuring SIRT1 level and its activity.

\section{Measurement of fasting serum glucose:}

The serum glucose was assessed by the method adopted by Trinder [14]

\section{Measurement of fasting serum insulin:}

Insulin concentrations were measured in previously frozen and thawed serum samples by enzymelinked immunosorbant assay (ELISA) using the Rat Insulin ELISA Kits (Linco research).

Assessment of serum triglycerides (TGs) and high-density lipoprotein (HDL):

TGs were measured by quantitative-enzymatic -colorimetric determination (Boehringer, Germany). HDL Cholesterol Assay Kit was purchased from Cell Biolabs, Inc., USA.

Detection of SIR T1 gene expression in skeletal muscle by Real time-Polymerase Chain Reaction (real time-PCR):

Tissue samples of all the studied groups were lysed and total RNA was isolated with RNeasy purification reagent (Qiagen, Valencia, CA). The purity of total RNA was measured with a spectro- 
photometer and the wave length absorption ratio (260/280nm) was between 1.8 and 2.0 for all preparations. Reverse transcription was carried out with reverse transcription reaction (Superscript II, Gibco Life Technologies, Grand Island, NY, USA). Real-time PCR amplification and analysis were performed using an Applied Biosystem with software version 3.1 (StepOneTM, USA). The reaction contained SYBR Green Master Mix (Applied Biosystems). The data were analyzed with the comparative cycle threshold (CT) method. The expression of $\beta$-actin mRNA was used as an internal control in all samples. The PCR primers sequence of SIRT 1 gene, Forward primer: 5'- GATCTCCCAGATCCTCAAGCC-3', Reverse primer: 5'CACCGAAGGAACTACCTGAT-3'

Determination of SIRT1 activity in skeletal muscle using SIRT1 Activity Assay Kit (Fluorometric)

SIRT1was extracted and purified from sample before use. First, H2O, SIRT1Assay Buffer, FluoroSubstrate Peptide and NAD were added to microtiter plate wells, then developer was added. Reactions were initiated by adding 5 othe Enzyme Sample, followed by read fluorescence intensity for 30 to 60 minutes at 1 to 2 minute intervals using micro titer plate fluoro meter with excitation at $340-360 \mathrm{~nm}$ and emission at $440-460 \mathrm{~nm}$. Measure and calculate the rate of reaction while the reaction velocity remains constant.

\section{Statistical methods:}

Data were coded and entered using the statistical package SPSS version 24. Data was summarized using mean, standard deviation. Comparisons between groups were done using analysis of variance (ANOVA) and multiple comparisons (Post Hoc test) for the quantitative variables. Correlations were done between variables using pearson correlation coefficient $(r) . p$-values less than 0.05 were considered as statistically significant.

\section{Results}

\section{BMI and serum blood glucose:}

Diabetic rats injected with STZ showed insignificant reduction in BMI in comparison to control group ( $p>0.05$ ), also, both exercise protocols resulted in insignificant increase in BMI relative to diabetic group $(p>0.05)$ (Table 1$)$.

Concerning fasting glucose, their levels were significantly increased in diabetic rats relative to control rats $(p<0.05)$. After exercise training for 1 month (D+Ex), fasting glucose levels were signif- icantly decreased relative to untrained diabetic rats. Remarkably, exercise protocol for one month preceding the induction of diabetes $(\mathrm{Ex}+\mathrm{D})$ achieved also an improvement in blood glucose levels $(p<0.05)$. These levels were insignificantly lower compared to exercise training after diabetes (Table 1).

\section{Serum lipids: triglycerides and $H D L$ :}

Parallel to hyperglycemia observed in diabetic group, (Fig. 1) showed a significant increase in serum triglycerides levels with significant decrease in HDL levels in diabetic group compared to control group $(p<0.05)$. On the contrary, Diabetic rats which undergo exercise for 1 month (D+Ex) showed an improvement in HDL levels $(p<0.05)$, with no significant difference in TG levels $(p>0.05)$. Both serum triglycerides and HDL were markedly preserved $(p<0.05)$ in rats undergo exercise training before induced diabetes $(E x+D)$. These data showed that exercise training before induced diabetes may be more beneficial than exercise after diabetes.

\section{SIRT level and activity:}

As observed in (Fig. 2), diabetic rats presented a significant lower SIRT1 level and activity in comparison to control ones $(p<0.05)$. Exercise for 1 month after diabetes $(\mathrm{D}+\mathrm{Ex})$ induced a significant improvement in serum SIRT1 level and activity in diabetic rats $(p<0.05)$. In addition, exercise training before induced diabetes $(\mathrm{Ex}+\mathrm{D})$ was significantly effective to counteract the reduction of SIRT 1 level and activity in diabetic group $(p<0.05)$. Both exercise groups failed to normalize the SIRT1 level and activity compared to control group, but, SIRT 1 level and activity values showed more improvement in rats undergo exercise training before diabetes. Correlations studies showed that SIRT level and activity were negatively correlated with serum glucose level respectively ( $r=-0.603, p<0.05$; $r=0.619, p<0.05)$. Also, SIRT1 level was negatively correlated with triglycerides $(r=-0.581$, $p<0.05)$ and positively correlated with HDL $(r=0.573, p<0.05)$ among studied groups (Fig. 3).

Table (1): Measurement of body mass index (BMI), fasting serum glucose among studied groups.

\begin{tabular}{lllll}
\hline Groups & Control & Diabetic & $\begin{array}{c}\text { Diabetes }+ \\
\text { exercise } \\
(\mathrm{D}+\mathrm{Ex})\end{array}$ & $\begin{array}{c}\text { Exercise+ } \\
\text { diabetes } \\
(\mathrm{Ex}+\mathrm{D})\end{array}$ \\
\hline Parameters & & & $0.42 \pm 0.06$ & $0.38 \pm 0.03$ \\
$\mathrm{BMI}\left(\mathrm{g} / \mathrm{cm}^{2}\right)$ & $0.44 \pm 0.06$ & $0.37 \pm 0.05$ & $0.42 \pm 0.10 \pm 2.48 \#$ \\
$\begin{array}{c}\text { Glucose } \\
(\mathrm{mmol} / \mathrm{L})\end{array}$ & $4.88 \pm 0.21$ & $13.02 \pm 2.20 *$ & $7.68 \pm 1.61 \#$ & $7.10 \pm$ \\
\hline
\end{tabular}

Values are represented as mean $\pm S D, n=10$.

$*: p<0.05$ statistically significant versus control.

$\#: p<0.05$ statistically significant versus diabetic group 

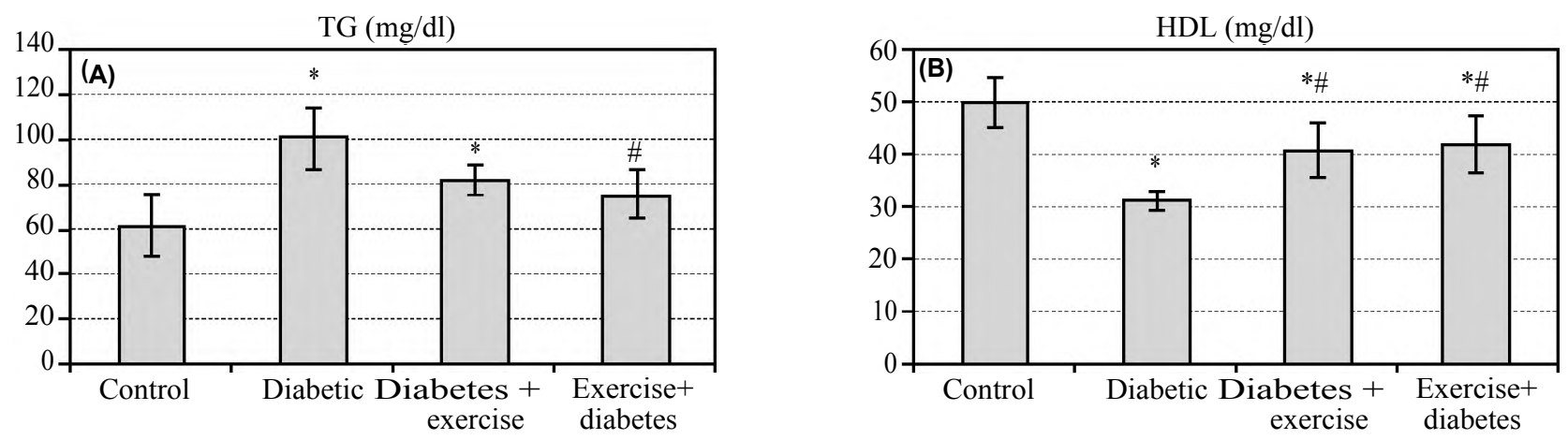

Fig. (1): (A) Serum triglycerides level (TG) in the studied groups, (B): High density lipoprotein (HDL) in the studied groups.

Each vertical bar represents the mean of 10 rats \pm SD. $\quad *: p<0.05$ statistically significant versus control.

$\#: p<0.05$ statistically significant versus diabetic group.
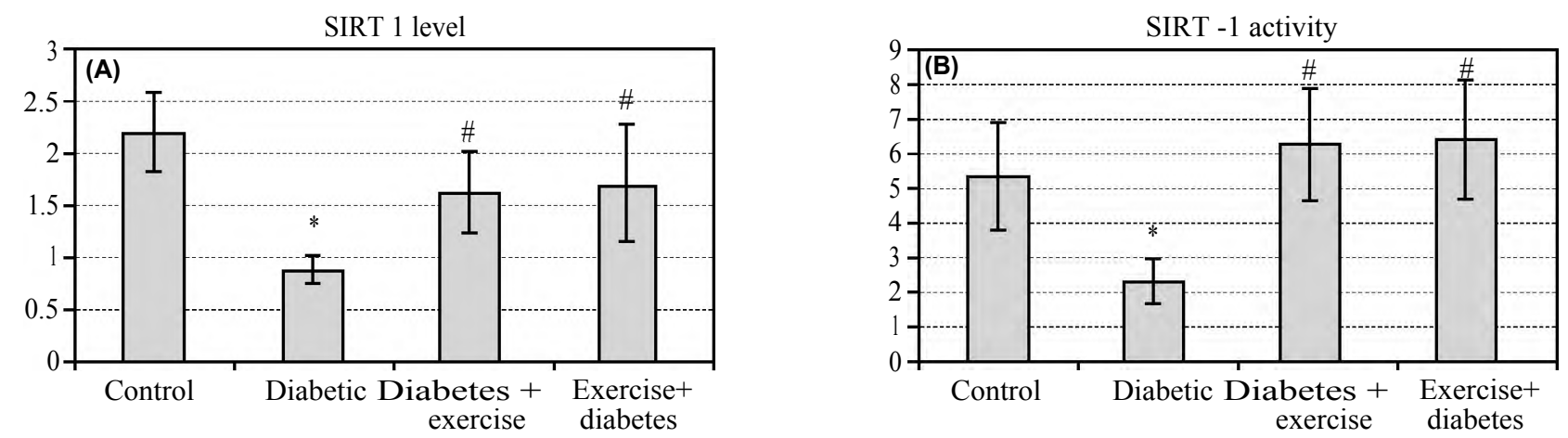

Fig. (2): (A) SIRT1 level in the studied groups, (B): SIRT 1 activity in the studied groups. Each vertical bar represents the mean of 10 rats $\pm \mathrm{SD}$.

$*: p<0.05$ statistically significant versus control. $\quad \#: p<0.05$ statistically significant versus diabetic group.
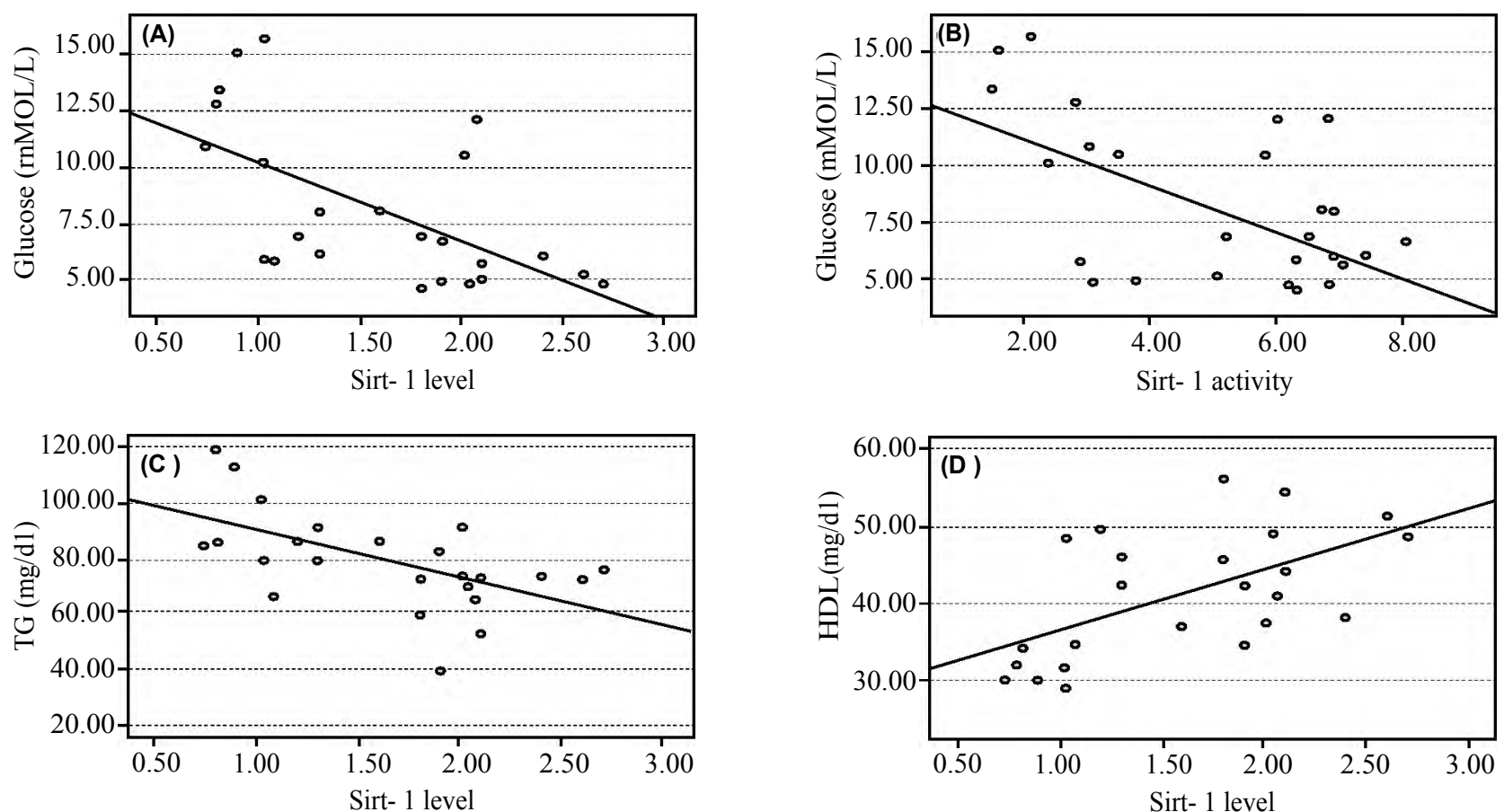

Fig. (3): Correlation (A): Between SIRT1 level and serum glucose, (B): Between SIRT1 activity and serum glucose, (C): Between SIRT1 level and triglycerides, (D): Between SIRT1 level and HDL among the studied groups. 


\section{Discussion}

Exercise has been considered to have important health benefits in diabetic peoples. The underlying molecular mechanisms of exercise-induced adaptations in diabetes remain undefined.

Therefore, the present work has been designed to test the hypotheses that the beneficial effect of exercise training on diabetes may be through reestablishing physiological activity of SIRT1, and compare between the effect of exercise before (as prophylactic measure) and after (as curative measure) induction of diabetes.

In our experiment, rats underwent exercise (swimming) for one month after induction of diabetes $(\mathrm{D}+\mathrm{EX})$ and one month before induction of diabetes $(\mathrm{EX}+\mathrm{D})$. With comparison to the diabetic group, results showed that fasting serum glucose levels were significantly decreased in both exercise regimens compared to hyperglycemia observed in diabetic group.

In agreement with our results, van Oort et al., [15] reported that single bout of 30min of exercise was shown to reduce the prevalence of hyperglycemia throughout the subsequent day. Yang et al. study [8] showed that training rats with weightbearing running, on a motor-driven treadmill markedly reduced the blood glucose level in type 1 diabetic rats after 6 weeks.

Several studies reported the effect of exercise on diabetes after induction of diabetes, with paucity of studies reporting the effect of exercise before development of diabetes.

Beside, our obtained data for BMI which showed a non-significant decrease in diabetic group compared to control group were in agreement with Haider et al., [16] who described that there was no significant effect of STZ-induced diabetes on body weight.

By studying the results of BMI in D+EX group, we found insignificant increase with comparison to diabetic group. Interestingly, BMI in $\mathrm{EX}+\mathrm{D}$ showed insignificant difference versus D+EX, suggesting no difference between the two exercise regimen and between diabetic rats as well. This may be explained by compensatory increase in food intake due to increase energy expenditure with exercise. Earlier investigators have demonstrated that exercise-treated diabetic patients usually show a compensatory increase of food intake due to decrease glucose utilization by the satiety center of the hypothalamus with failure to inhibit the feeding center [17]

While Körner et al., [18] opposing our results, suggested that the aerobic exercise training reduced the body weight in trained rats with metabolic syndrome relative to their sedentary controls due to increase in the metabolic rate with decreased fat content.

Dyslipidemia is a common feature that causes morbidity and mortality in diabetic patients. Accordingly, we found a significant elevation of TG and significant reduction of HDL in diabetic rats versus the control group.

We also reported that D+EX group showed improvement in dyslipidemia presented by significant lower TG but HDL increased insignificantly. Remarkably, EX+D group results showed better results as both HDL and TG improved significantly in relation to diabetic group.

Leon \& Sanchez, [19] in agreement with our results conducted a meta-analysis showed increased HDL cholesterol and decreased triglyceride levels after 12 weeks of aerobic exercise.

On the other hand, randomized controlled clinical trials used aerobic and resistance exercise and reported limited beneficial results, mainly in elderly [20] and middle-aged people [21]. Beside, Lee, [22] suggested a combined folk dance and resistance training protocol for 24 weeks found no significant effect on lipid profile.

Sangwoon et al., [23] concluded that comparing with sedentary individuals, those who are regularly physically active and fit have been shown to have better lipid profiles including TG and HDL subfractions and lower amounts of visceral fat at a given level of body weight.

How exactly exercise exerts its beneficial effects in diabetes is not fully understood; however, skeletal muscle is believed to play a vital role.

In this issue, Colberg et al., [4] reported that exercise training alleviate insulin resistance and improve glucose tolerance in type 2 DM. Yang et al., [24] and Banks et al., [25] suggested that changes in muscle morphology with increase in capillary density following training may also be important in insulin sensitivity. Leon \& Sanchez, [19] reported the improving effect of exercise on dyslipidemia associating diabetes.

Complementary to these studies, in the current study, we examined the potential role of SIRT in 
diabetic rats following exercise as one of the proposed mechanisms. Several studies examined the role of SIRT in exercise, but its role in relation to diabetes still under estimation.

In rats, SIRT1 expression is increased in skeletal muscle after both acute endurance exercise and chronic exercise [26]. In accordance, our results showed that the effect of exercise on both SIRT1 level and activity was obvious as they were significantly increased in $(\mathrm{D}+\mathrm{EX})$ and $(\mathrm{EX}+\mathrm{D})$ groups compared to diabetic group. Results from $(\mathrm{EX}+\mathrm{D})$ group insignificantly increased compared to the $\mathrm{D}+\mathrm{EX}$ group.

Interestingly, our results concluded that there were strong negative correlations between SIRT1 level, activity and glucose level as well as SIRT1 level was negatively correlated with TG and positively correlated with HDL level among all groups. These results may guide us to say that SIRT1 level and activity play important role in glucose and lipid homeostasis during exercise.

\section{Conclusion and recommendations:}

Both exercise regimens may be beneficial in improving hyperglycemia and hyperlipidemia associating diabetes, however, pre diabetes exercise appears to be more effective than post diabetes exercise and nearly normalize them, yet this difference is insignificant. Activation of SIRT may be incorporated in the mechanisms by which exercise induced its beneficial effect on diabetes. Therefore, we recommend the use of SIRT1 agonists as new promising approaches for prevention of diabetes in pre-diabetics and treating diabetics even those with complication. Also, further research in other life style modifications and their relation to SIRT1 may offer potential solutions for diabetes and its complications.

\section{Declaration of interest.}

The authors declare no conflict of interest.

There is no source of funding.

\section{References}

1- SUN Z., LIU L., LIU N., et al.: Muscular response and adaptation to diabetes mellitus. Front Biosci., 13: 47654794, 2008.

2- DYSON P., KELLY T., DEAKIN T., et al.: Recommendations Diabetes UK evidence-based nutrition guidelines for the prevention and management of diabetes. Diabet Med., 28 (11): 1282-8, 2011.

3- GRUNDY S.M., GARBER A., GOLDBERG R., et al. Prevention Conference VI: Diabetes and Cardiovascular Disease: Writing Group IV: Lifestyle and medical man- agement of risk factors. Circulation, 105 (18): e153-e158, 2002.

4- COLBERG S.R., SIGAL R.J., FERNHALL B., et al.: Exercise and type 2 diabetes: The American College of Sports Medicine and the American Diabetes Association: joint position statement. Diabetes Care, 33 (12): 2692-6, 2010.

5- GURD B.: Deacetylation of PGC-1 $\alpha$ by SIRT1: importance for skeletal muscle function and exercise-induced mitochondrial biogenesis. Appl. Physiol. Nutr. Metab., 36 (5): 589-597, 2011.

6- WILLIAMS C.B. and GURD B.J.: Skeletal muscle SIRT1 and the genetics of metabolic health: Therapeutic activation by pharmaceuticals and exercise. Application of Clinical Genetics, 5: 81-91, 2012.

7- SAFDAR A., HAMADEH M., KACZOR J., et al.: Aberrant mitochondrial homeostasis in the skeletal muscle of sedentary older adults. PloS One, 5 (5): e10778, 2010.

8- YANG J., SUN L., FAN X., et al.: Effect of exercise on bone in poorly controlled type 1 diabetes mediated by the actriib/smad signaling pathway. Experimental and therapeutic medicine, 16: 3686-3693, 2018.

9- ABU ABEELEH M., ZUHAIR B. and KHALED R.: Induction of Diabetes Mellitus in Rats Using IntraperitonealStreptozotocin: A Comparison between 2 Strains of Rats. European Journal of Scientific Research, 32 (3): 398-402, 2009.

10- GOBATTO C.A., DE MELLO M.A., SIBUYA C.Y., et al.: Maximal lactate steady state in rats submitted to swimming exercise. Comp. Biochem. Physiol. A Mol. Integr. Physiol., 130 (1): 21-7, 2001.

11- BOTEZELLI J.D., MORA R.F., DALIA R.A., et al.: Exercise counteracts fatty liver disease in rats fed on fructose-rich diet. Lipids in Health and Disease, 9: 116, 2010 .

12- SEVILLANO J., CASTRO J., BOCOS C., et al.: Role of insulin receptor substrate-1 serine 307phosphorylation and adiponectin in adipose tissue insulin resistance in late pregnancy. Endocrinology, 148 (12): 5933-5942, 2007.

13- NOVELLI E.L., DINIZ Y.S., GALHARDI C.M., et al.: Anthropometrical parameters and markers of obesity in rats. Lab Anim., 41 (1) 111-9, 2007.

14- TRINDER P.: Evaluation of Trinder glucose oxidase method. Clin. Chem., 21/12, 1754-1760, 1975.

15- VAN OORT M.M., VAN DOORN J.M., HASNAOUI M.E., et al.: Effects of AMPK activators on the subcellular distribution of fatty acid transporters CD36 and FABPpm. Arch. Physiol. Biochem., 115 (3): 137-146, 2009.

16- HAIDER S., AHMED S., TABASSUM S., et al.: Streptozotocin-induced insulin deficiency leads to development of behavioral deficits in rats. Acta. Neurol. Belg., 113 (1): 35-41, 2013.

17- WING R.R., GOLDSTEIN M.G., ACTON K.J., et al.: Behavioral science research in diabetes: Lifestyle changes related to obesity, eating behavior, and physical activity. Diabetes Care, 24 (1): 117-123, 2001. 
18- KÖRNER A., KIESS W., STUMVOLL M., et al.: Polygenic contribution to obesity: genome-wide strategies reveal new targets. Front Horm. Res., 36: 12-36, 2008.

19- LEON A. and SANCHEZ O.: Response of blood lipids to exercise training alone or combined with dietary intervention. Med. Sci. Sports Exerc., 33 (6 Suppl) S502-15, 2001.

20- BOARDLEY D., FAHLMAN M., TOPP R., et al.: The impact of exercise training on blood lipids in older adults. Am. J. Geriatr. Cardiol., 16: 30-5, 2007.

21- PARK S., PARK J., KWON Y., et al.: The effect of combined aerobic and resistance exercise training on abdominal fat in obese middle-aged women. J. Physiol. Anthropol., 22 (3): 129-35, 2003.

22- LEE K.: Effects of exercise program on body composition, physical fitness and lipid metabolism for middle-aged obese women. Taehan Kanho Hakhoe Chi., 35: 1248-57, 2005.

23- SANGWOON C., HONGWEI Y., SAMUEL C., et al.: Regulation of SIRT1 in cellular functions: role of polyphenols. Arch. Biochem. Biophys., 501 (1): 79-90, 2010.

24- YANG H., YANG T., BAUR J.A., et al.: Nutrient-sensitive mitochondrial NAD+ levels dictate cell survival. Cell, 130 (6): 1095-1107, 2007.

25- BANKS A., KON N., KNIGHT C., et al.: SirT1 gain of function increases energy efficiency and prevents diabetes in mice. Cell Metab., 8: 333-341, 2008.

26- SUWA M., NAKANO H., RADAK Z., et al.: Endurance exercise increase the SIRT 1 and peroxisome proliferator activated-receptor gamma coactivator-1alpha protein expression in rat skeletal muscle. Metabolism, 57: 986998, 2008.

\section{تأثير التفاعل بين التمارين الرياضية وبروتين السيرت رقهم واحل

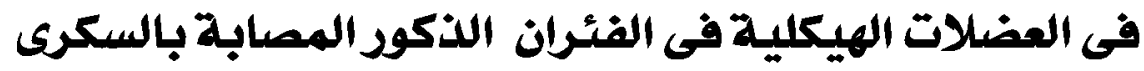

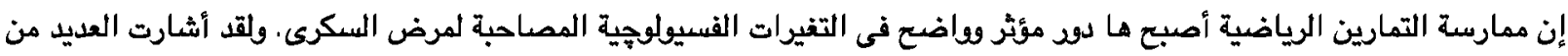

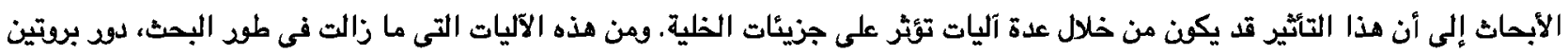

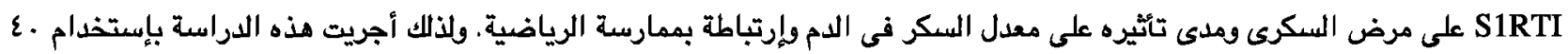

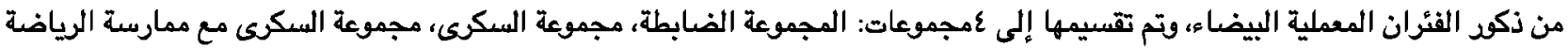

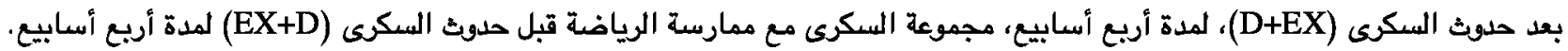

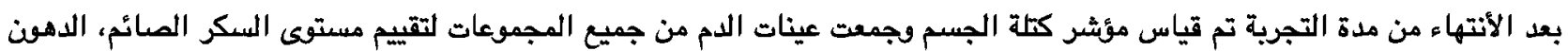

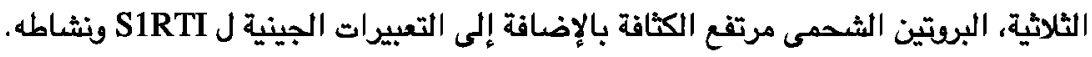

كشفت النتائج عن إنحفاض ملحوظ فى مستوى السكر الصائم والدهن الثلاثية مع إرتقاع ملحوظ فى البروتين الشحمى مرثفع الكثافة

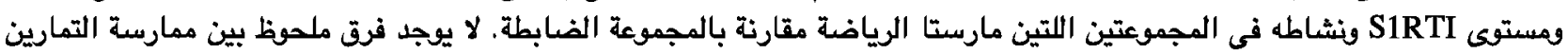
الرياضية قبل وبعد مرض السكرى.

وبدارسة إرتباط النتائج، تبين أن زيادة التعبير الجينى ل S1RTI ونشاطه حدث بالتوازى مع نقص مستوى السكر الصائم، والدهون الثُاثية، وزيادة البروتين الثحمى مرتفع الكثافة.

يمكننا أن نستنتج أن دور ممارسة الرياضدة فى تحسين حالة مرض السكرى أن يككن من خلال زيادة التعبير الجينى ل S1RTI وزيادة 